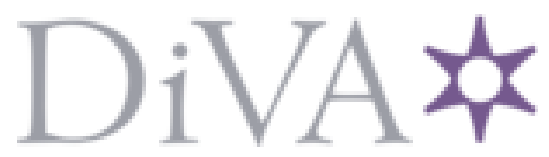

http://www.diva-portal.org

\title{
Postprint
}

This is the accepted version of a paper presented at North American Power Symposium (NAPS).

Citation for the original published paper:

nasri, a., Eriksson, R., Ghandhari, M. (2013)

Suitable placements of multiple FACTS devices to improve the transient stability using trajectory sensitivity analysis.

In:

N.B. When citing this work, cite the original published paper.

Permanent link to this version:

http://urn.kb.se/resolve?urn=urn:nbn:se:kth:diva- 139540 


\section{Suitable Placements of Multiple FACTS Devices to Improve the Transient Stability Using Trajectory Sensitivity Analysis}

\author{
Amin Nasri \\ Electric Power System Department \\ KTH Royal Institute of Technology \\ Stockholm, Sweden \\ Email: amin.nasri@ee.kth.se
}

\author{
Robert Eriksson \\ Electric Power System Department \\ KTH Royal Institute of Technology \\ Stockholm, Sweden \\ Email: robert.eriksson@ee.kth.se
}

\author{
Mehrdad Ghandhari \\ Electric Power System Department \\ KTH Royal Institute of Technology \\ Stockholm, Sweden \\ Email: mehrdad.ghandhari@ee.kth.se
}

\begin{abstract}
Trajectory sensitivity analysis (TSA) is used as a tool for suitable placement of multiple series compensators in the power system. The goal is to maximize the benefit of these devices in order to enhance the transient stability of the system. For this purpose, the trajectory sensitivities of the rotor angles of the most critical generators with respect to the reactances of transmission lines are calculated in the presence of the most severe faults. Based on the obtained trajectory sensitivities, a method is proposed to determine how effective the series compensation of each transmission line is for improving the transient stability. This method is applied to the Nordic-32 test system to find the priorities of the transmission lines for installation of several series compensators. Simulation with industrial software shows the validity and efficiency of the proposed method.
\end{abstract}

\section{INTRODUCTION}

Transient stability is the ability of power system to keep its synchronism when a large disturbance, such as three phase short circuit, occurs in the system. Nowadays, deregulation in electricity markets, increasing electricity demands and high penetration of renewable energy sources on one hand and economic and environmental constraints on installing new transmission lines and building new power plants on the other hand, have pushed the existing transmission systems to be operated close to their critical conditions. So, there is a higher risk of transient instability in today's heavy-loaded and interconnected power systems.

Series Flexible AC Transmission Systems (FACTS) devices such as Thyristor Controlled Series Compensation (TCSC) can have a significant impact on operational flexibility and controllability of the power system. They can dynamically change the total reactance of transmission lines and control the power flow through lines in a way to increase the transient stability margins and make the system more secure. Since the impact of these compensators on the system's stability is strongly dependent on their locations, there is a great need for developing an analytical tool to provide useful information to the system planners regarding the best possible installation locations of them. In this paper, an effective tool is developed to identify the most suitable placement of multiple series compensators for improving transient stability.
Assessment of rotor angle stability is essential to study the dynamic behaviour of the power system. Time domain simulation is the traditional way for transient stability assessment which has two main disadvantages, namely time-consuming computation requirement and incapability to provide any information regarding the stability margin [1]. The other method which has been widely used for this purpose is transient energy function (TEF) method, see [2], [3], and [4]. The significant advantage of this method is its capability to provide a stability index [1]. Several methodologies have been proposed based on the sensitivity of TEF to determine the effectiveness of FACTS devices to improve system's transient stability, see [5], and [6]. Despite all the advantages of the TEF based methods, the main shortcoming of them is their high complexity in the following situations: (i) considering differential-algebraic equation (DAE) models of power systems, (ii) dealing with the detailed models of the system's components, (iii) when a number of system's parameters have to be taken into account for the sensitivity analysis [7].

Applications of trajectory sensitivity analysis (TSA) have been introduced as an alternative to overcome the mentioned shortcoming of the TEF based method [8]. Reference [9] has used TSA to calculate the critical values of some power system's parameters such as fault clearing time, mechanical input power of generators, etc. Their method is based on the computation of the norm of trajectory sensitivities of rotor angles and speeds of generators with respect to the parameters of interest. Reference [10] has discussed the application of TSA to power systems containing series and shunt compensators. A transient stability index has been introduced based on the numerical formulation of TSA and has been calculated for the power system with different locations of series and shunt compensators. Using numerical formulation of TSA, considering the compensators' models in the study and simulating the power system for all the possible locations of compensators has caused high computational burden in their proposed method. Reference [7] has developed a multi-parameter trajectory sensitivity approach to find the best locations of series compensators in order to improve 
the transient stability. An index of proximity to instability has been determined based on the norm of the trajectory sensitivities of the rotor angles and the speeds of generators with respect to the transmission line susceptances. Using the analytical formulation of TSA in the last mentioned paper and also in the current article, the cumbersome computational process becomes much simpler compared to the methodologies based on the numerical estimation of TSA. The latter will be explained later.

In this paper, a novel method is proposed based on TSA to determine the most appropriate places of series compensations to amend the transient stability of power system. Trajectory sensitivities of the rotor angles of critical generators are computed directly to the reactance of different transmission lines considering the most severe contingencies. The obtained sensitivity curves show perfectly the effect of each transmission line's reactance on the rotor angle's trajectory of each generator, and can be used to identify the the most effective locations to install series compensators for transient stability improvement. The proposed methodology also shows why putting series compensator in one transmission line has a positive and in the other line has a negative effect on the system's stability. The way to carry out the multiple placement is coordinated since the previously installed FACTS devices are considered for each new compensator's allocation. Finally, the authors believe that the proposed method is computationally efficient since analytical formulation of TSA are used and suitable system's parameters are chosen. The system under considerations is Nordic-32 test system.

\section{POWER SYSTEM MODELING AND TRAJECTORY SENSITIVITY ANALYSIS}

As explained comprehensively by [11], power systems can be modeled by the following differential algebraic equations

$$
\begin{gathered}
\underline{\dot{x}}=\underline{f}(\underline{x}, y) \\
0=\left\{\begin{array}{lr}
g^{-}(\underline{x}, y) & s(\underline{x}, y)<0 \\
g^{+}(\underline{x}, y) & s(\underline{x}, y)>0
\end{array}\right. \\
\underline{x}=\left[\begin{array}{l}
x \\
\lambda
\end{array}\right] \quad \underline{f}=\left[\begin{array}{l}
f \\
0
\end{array}\right] \\
\underline{x}\left(t_{0}\right)=\underline{x}_{0}, y\left(t_{0}\right)=y_{0}
\end{gathered}
$$

where $x$ is a vector containing the dynamic states, $y$ is a vector of algebraic states and $\lambda$ is a vector of system parameters. Rotor angles of the generators $(\delta)$, magnitude and angle of bus voltages and reactances of the transmission lines are the examples of the dynamic states, algebraic states and parameters of the power system, respectively. $x_{0}$ and $y_{0}$ are the initial conditions of dynamic and algebraic states. Function $f$ is the set of differential equations which model the dynamics of equipments such as generators. Events such as a three phase short circuit fault occurs when $s(\underline{x}, y)=0$. The algebraic equations $g$ consist of the network equations based on Kirchhoff's current law, i.e. the sum of all current (or powers) flowing into each bus must be equal to zero, $g^{-}$and $g^{+}$show the algebraic equations before and after occurrence of events.

To calculate the trajectory sensitivities analytically, the derivatives of (1), (2) are calculated with respect to $\underline{x}_{0}$

$$
\begin{gathered}
\underline{\dot{x}}_{\underline{x}_{0}}=\underline{f}_{\underline{x}}(t) \underline{x}_{\underline{x}_{0}}+\underline{f}_{y}(t) y_{\underline{x}_{0}} \\
0=g_{\underline{x}}(t) \underline{x}_{\underline{x}_{0}}+g_{y}(t) \underline{y}_{0}
\end{gathered}
$$

The system is assumed to be away from the events. The initial conditions for $\underline{x}_{\underline{x}_{0}}$ and $\underline{y}_{\underline{x}_{0}}$ are obtained by differentiating (4) with respect to $\underline{x}_{0}$. It is clear that the initial value for the trajectory sensitivities of dynamic states is an identity matrix. Using this identity matrix, the initial values for the trajectory sensitivities of algebraic states can be also computed from (6).

$$
\underline{x}_{\underline{x}_{0}}\left(t_{0}\right)=I, \quad y_{\underline{x}_{0}}\left(t_{0}\right)=-\left(g_{y}\left(t_{0}\right)\right)^{-1} g_{\underline{x}_{0}}\left(t_{0}\right)
$$

$\underline{f}_{x}, \underline{f}_{y}, g_{\underline{x}}$ and $g_{y}$ are time varying functions which are calculated along the system trajectories. When an event occurs in the system, jump condition should be derived for computation of the trajectory sensitivities which is fully described by [11]. To find the trajectory sensitivities, the DAEs (1)-(2) and (5)(6) will be solved simultaneously with the initial conditions described above using trapezoidal integration technique.

To calculate the trajectory sensitivity of state variable $x$ to the parameter $\lambda$ with a numerical formulation of TSA, a small perturbation of $\triangle \lambda$ over the nominal parameter $\lambda_{0}$ should be considered such that $\lambda=\lambda_{0}+\triangle \lambda$. So, the numerical estimation of sensitivity is defined as

$$
x_{\lambda}=\frac{x(\lambda)-x\left(\lambda_{0}\right)}{\triangle \lambda}
$$

\section{TRANSIENT STABILITY ASSESSMENT USING TRAJECTORY SENSITIVITY ANALYSIS}

The result of transient instability appears in the form of increasing rotor angles of some generators which leads to their loss of synchronism with other generators. So, monitoring angular swings of generators (dynamic states $\delta$ ) could be one way to check the transient stability. To improve the transient stability, power system parameters can be controlled (if applicable) in a way to have positive effects on the variation of rotor angles of generators when the system is subjected to a fault and prevent power system from being unstable. Reactance of transmission line is one of these parameters which can have a considerable effect on the stability of power system with controlling power flows in the transmission lines. Nowadays with the presence of FACTS devices, it is possible to control the reactance of transmission lines and improve transient stability of power system. Due to heavy cost of these devices, it is not economical to install several series compensators in a power system, and instead, the optimal locations for installation of these devices should be determined. In this paper, TSA is used to determine the trajectory sensitivities of rotor angles of generators to the reactances of transmission lines. So, the matrices of power system parameters and trajectory sensitivities of dynamical states for this study are as follows

$$
\lambda=\left[x_{L_{1}} x_{L_{j}} \ldots x_{L_{n_{l}}}\right]
$$




$$
\frac{\partial \delta}{\partial \lambda}=\left[\begin{array}{cccc}
\frac{\partial \delta_{1}}{\partial x_{L_{1}}} & \frac{\partial \delta_{1}}{\partial x_{L_{k}}} & \cdots & \frac{\partial \delta_{1}}{\partial x_{L_{n}}} \\
\frac{\partial \delta_{2}}{\partial x_{L_{1}}} & \frac{\partial \delta_{2}}{\partial x_{L_{k}}} & \cdots & \frac{\partial \delta_{2}}{\partial x_{L_{n_{l}}}} \\
\cdot & \cdot & \cdot & \cdot \\
\cdot & \cdot & \cdot & \cdot \\
\frac{\partial \delta_{n}}{\partial x_{L_{1}}} & \frac{\partial \delta_{n}}{\partial x_{L_{k}}} & \cdots & \frac{\partial}{\partial x_{L_{n}}}
\end{array}\right]
$$

where $\delta_{i}$ is the rotor angle of the $i^{\text {th }}$ generator, $n$ is the number of generators, $x_{L_{k}}$ is the reactance of the $\mathrm{k}^{\text {th }}$ transmission line, and $n_{l}$ is the number of lines. The matrix (10) is a part of solution to the (5) described in Section II.

\section{APPROPRIATE PLACEMENT OF SERIES COMPENSATORS TO IMPROVE THE TRANSIENT STABILITY}

The proposed algorithm for appropriate placement of multiple series compensators to improve transient stability is as follows

1) Most severe faults with the following clearing times are selected.

$$
t_{c l_{i}}=t_{c c_{i}}-\varepsilon
$$

where $t_{c l_{i}}$ and $t_{c c_{i}}$ are the clearing time and critical clearing time (CCT) of the $i^{t h}$ fault respectively, and $\varepsilon$ is a positive small number (1 $\mathrm{ms}$ in this paper).

2) For each fault:

2.1) (1)-(2) and (5)-(6) are solved simultaneously using the mentioned $\lambda$ vector and all the dynamic and algebraic variables and their trajectory sensitivities with respect to the parameter vector $\lambda$ are calculated. Simulation time does not need to be so long (first swing only).

2.2) Based on the obtained data, the rotor angles $(\delta)$ of the generators are depicted in center of inertia $(C O I)$ reference and only the most critical generators (close to instability) will be selected. These generators are divided into $A$ and $D$ groups depending if their rotor angles $(\delta)$ after fault occurrence are accelerating or decelerating, respectively.

2.3) The matrix of trajectory sensitivities of dynamical states (10) corresponding to the most critical generators is obtained and trajectory sensitivities of $\delta_{i}$ with respect to the reactances of different transmission lines are computed.

2.4) A normalized index of trajectory sensitivity $\left(\widehat{S}_{z k}\right)$ is calculated for each transmission line which shows the effectiveness of that line for improving the transient stability. The definition of $\widehat{S}_{z k}$ is as follows

$$
\begin{gathered}
\widehat{\mathrm{S}}_{\mathrm{zk}}=\frac{\mathrm{S}_{\mathrm{zk}}}{\mathrm{S}_{\mathrm{zk}}^{\max }} \cdot \frac{\mathrm{x}_{\mathrm{L}_{\mathrm{k}}}}{\mathrm{x}_{\mathrm{L}_{\mathrm{k}}}^{\max }} \quad \forall \mathrm{k} \in \mathrm{L}, \forall \mathrm{z} \in \mathrm{Z} \\
\mathrm{S}_{\mathrm{zk}}=\mathrm{PP}_{\mathrm{T}}\left\{\mathrm{M}_{\mathrm{A}}^{-1} \sum_{\mathrm{i} \in \mathrm{A}} \mathrm{M}_{\mathrm{i}} \frac{\partial \delta_{\mathrm{i}}}{\partial \mathrm{x}_{\mathrm{L}_{\mathrm{k}}}}-\mathrm{M}_{\mathrm{D}}^{-1} \sum_{\mathrm{j} \in \mathrm{D}} \mathrm{M}_{\mathrm{j}} \frac{\partial \delta_{\mathrm{j}}}{\partial \mathrm{x}_{\mathrm{L}_{\mathrm{k}}}}\right\} \\
\forall k \in L, \forall z \in Z \\
M_{A}=\sum_{i \in A} M_{i}, M_{D}=\sum_{j \in D} M_{j}
\end{gathered}
$$

where

$\widehat{\mathrm{S}}_{\mathrm{zk}}$ : is the normalized index of trajectory sensitivity corresponding to each transmission line.

$\mathrm{S}_{\mathrm{zk}}^{\max }$ : is the maximum value of $\mathrm{S}_{\mathrm{zk}}$ among all the transmission lines for fault $z$.

$x_{L_{k}}^{\max }$ : is the maximum value of $x_{L_{k}}$ among all the transmission lines.

$\mathrm{S}_{\mathrm{zk}}$ : is the index of trajectory sensitivity corresponding to each transmission line.

$P P_{T}$ : gives the peak-to-peak value of a function for the period $T$.

$M_{i}$ : is the inertia of $i^{t h}$ generator.

$A$ : is the set of critical generators which accelerate after fault occurrence.

$D$ : is the set of critical generators which decelerate after fault occurrence.

$L:$ is the set of transmission lines.

$Z$ : is the set of selected severe faults.

$M_{A}$ : is sum of inertia constants of the generators belonging to the set $A$.

$M_{D}$ : is sum of inertia constants of the generators belonging to the set $D$.

$\partial \delta_{i} / \partial x_{L_{k}}$ : is the trajectory sensitivity of rotor angle of $i^{\text {th }}$ generator with respect to the reactance of $k^{t h}$ transmission line.

3) The transmission lines with the greatest positive values of total trajectory sensitivity indices $\left(\mathrm{TS}_{\mathrm{k}}\right)$ are the most appropriate places to install series compensator for improving the transient behavior of the power system corresponding to $p(z)$

$$
\mathrm{TS}_{\mathrm{k}}=\sum_{\mathrm{z} \in \mathrm{Z}} \mathrm{p}(\mathrm{z}) \widehat{\mathrm{S}}_{\mathrm{zk}} \quad \forall \mathrm{k} \in \mathrm{L}, \forall \mathrm{z} \in \mathrm{Z}
$$

where

$\mathrm{TS}_{\mathrm{k}}$ : is the total trajectory sensitivity index corresponding to each transmission line.

$p(z)$ : is a function which determines the degree of importance of each fault. This function could be a combination of the occurrence probability, degree of severity and etc.

4) To find the appropriate location to install the next series compensator, step (1)-(3) are repeated with the following changes 
4.1) The system under study is modified considering all the previously specified series compensators installed at their best locations.

4.2) The transmission lines which already have the series compensator are removed from the list of candidate locations to put the new device.

4.3) Set of selected faults and their corresponding $p(z)$ are updated considering the installed devices.

\section{Simulation AND Results}

The test system used in this study is the Cigre Nordic 32bus test system. The system is shown in Fig. 4. The system data is taken from [12]. The Classical model is used for the generators. Table I shows list of the selected faults which are applied to this test system. When a fault occurs in the system, critical generators are determined and divided to two transient groups as mentioned in Section 4.2.2. Fig. 2 shows the rotor angles of generators in center of inertia reference for fault 3 . It is clear that the generators 11 and 12 which are the most critical ones (close to instability) are both accelerating. So, the $A$ and $D$ sets for this fault are as follows

$$
A=\{G 11, G 12\}, D=\{\}
$$

To find the value of $S_{z k}$ from (13), trajectory sensitivities of rotor angle of $G 11$ and $G 12$ should be depicted. The trajectory sensitivities of $\delta_{11}$ with respect to the reactances of different transmission lines are shown in Fig. 3. This figure shows that L27-12, $\widehat{L} 12$ - 11, L8-27 and L26-12 have the largest positive peak to peak values and L25-9 has the most negative value of trajectory sensitivities. The symbols $\mathrm{Li}-\mathrm{j}$ and $\widehat{\mathrm{Li}}-\mathrm{j}$ determines the first and second transmission line connecting bus $i^{t h}$ to $j^{t h}$.

It is important to understand the meaning of the peak to TABLE I

LIST OF SELECTED FAULTS - NORDIC 32-BUS TEST SYSTEM

\begin{tabular}{c|c|c}
\hline Name & Fault Location & Clearing Time \\
\hline Fault 1 & Line $4-21$ close to bus 4 & $166 \mathrm{~ms}$ \\
\hline Fault 2 & Line $6-22$ close to bus 6 & $220 \mathrm{~ms}$ \\
\hline Fault 3 & Line $12-11$ close to bus 11 & $188 \mathrm{~ms}$ \\
\hline Fault 4 & Line $17-18$ close to bus 17 & $238 \mathrm{~ms}$ \\
\hline Fault 5 & Line $24-9$ close to bus 9 & $265 \mathrm{~ms}$ \\
\hline
\end{tabular}

peak values and their signs after occurring faults. It can be written based on the trajectory sensitivities

$$
\triangle \delta_{11} \approx\left(\frac{\partial \delta_{11}}{\partial x_{L_{i}}}\right) \triangle x_{L_{i}}
$$

As series capacitor is going to be installed in the transmission lines, $\triangle x_{L_{i}}$ will be negative and since $\delta_{11}$ will also increase rapidly after occurring fault which is visible from Fig. 2, the value of trajectory sensitivity of $\delta_{11}$ with respect to the reactance of the transmission line should be positive to make $\triangle \delta_{11}$ negative, decrease $\delta_{11}$, and enhance the transient stability of the system. Thus, absolute peak to peak value of trajectory sensitivity shows how sensitive $\delta_{11}$ is to the reactances of the transmission lines and its sign determines if putting series capacitor in that line has positive or negative effect on the transient stability. Since, generator 12 also has a critical condition for this fault, the same analysis has been done for $\delta_{12}$

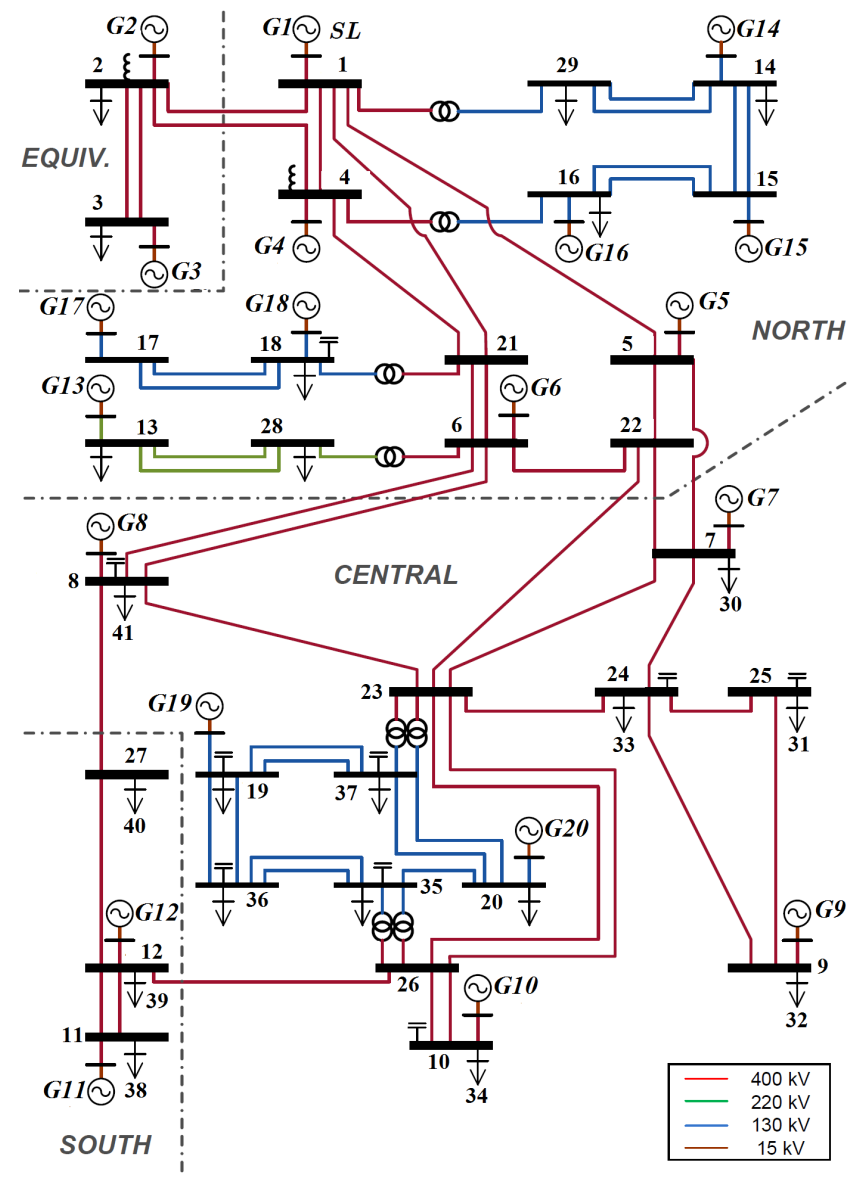

Fig. 1. The Cigre Nordic 32-bus test system

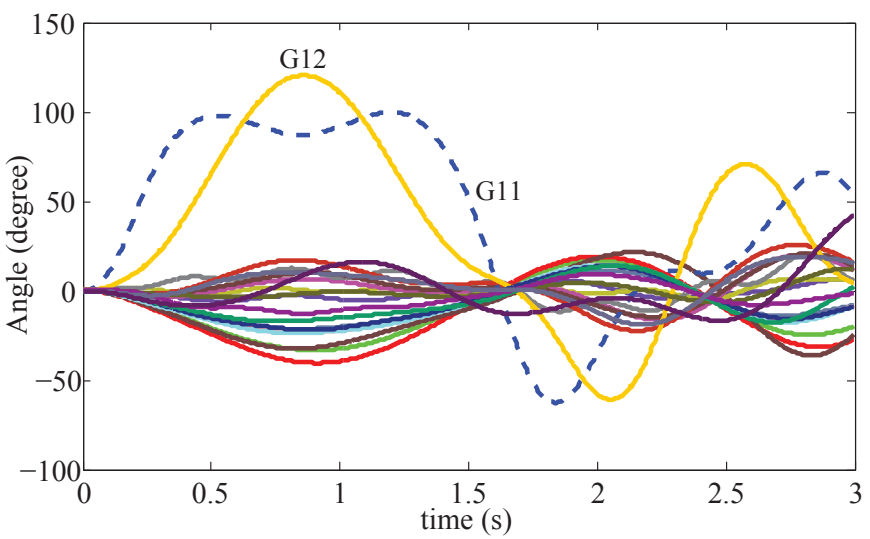

Fig. 2. Rotor angles of generators in center of inertia reference for fault 3

as well, and the values of $S_{\mathrm{zk}}$ have been calculated based on (13). Then, $\widehat{\mathrm{S}}_{\mathrm{zk}}$ which is the normalized index of trajectory sensitivity has been calculated for each transmission lines according to (12). The term $x_{L_{k}} / x_{L_{k}}^{\max }$ in (12) is because of the practical limitation of each line and gives a higher weight to those lines with larger values of reactance and therefor larger possible amount of series compensation. The similar calculations have been done for the other selected faults, and due to space limitation, only the most important results are given in Table 2 and 3. Table 2 shows the $A$ and $D$ sets for 


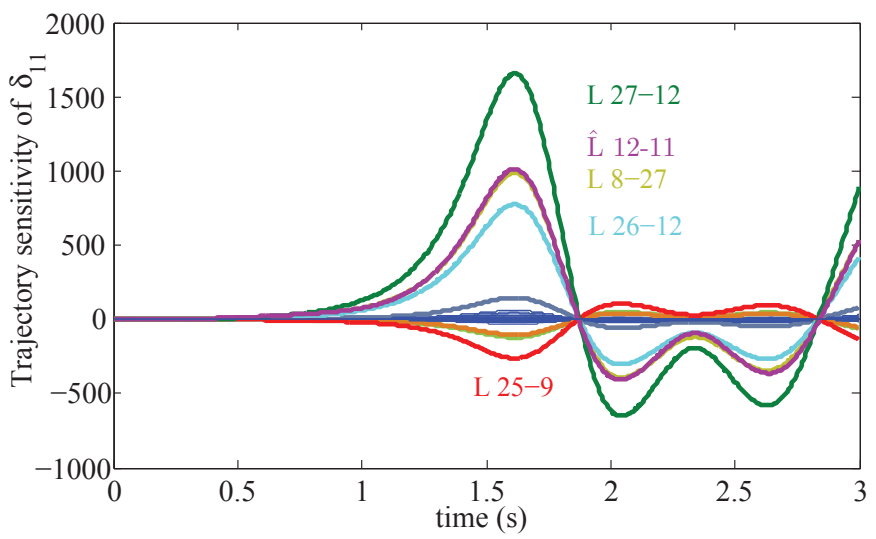

Fig. 3. Trajectory sensitivities of $\delta_{11}$ with respect to the reactances of different transmission lines for fault 3

different fault scenarios. As it can be seen in Table 3, for each fault, the first three priorities to install the series device and also the worst one are shown. It should be noted that these values are normalized indices of trajectory sensitivities $\widehat{\mathrm{S}}_{\mathrm{zk}}$ and are different from $\mathrm{S}_{\mathrm{zk}}$ values. For instance, in case of fault 3, Fig. 3 depicts that L27-12 is the most sensitive place because it has the highest peak to peak value $\left(\mathrm{S}_{\mathrm{zk}}\right)$, but the most appropriate place to install the device is L2612 (highest $\widehat{\mathrm{S}}_{\mathrm{zk}}$ according to the Table 3). This is because of higher reactance of L26-12 compared to L27-12.

TABLE II

SETS $A$ AND $D$ FOR DIFFERENT FAULTS

\begin{tabular}{c|c|c|c|c|c}
\hline Fault & 1 & 2 & 3 & 4 & 5 \\
\hline Set $A$ & $\{\mathrm{G} 4\}$ & $\{\mathrm{G} 13\}$ & $\{\mathrm{G} 11, \mathrm{G} 12\}$ & $\{\mathrm{G} 17\}$ & $\{\mathrm{G} 9\}$ \\
\hline Set $D$ & \{\} & \{\} & \{\} & \{\} & \{\} \\
\hline
\end{tabular}

TABLE III

NORMALIZED INDICES OF TRAJECTORY SENSITIVITIES $\widehat{\mathrm{S}}_{\mathrm{zk}}$

\begin{tabular}{c|c|c|c|c}
\hline$\widehat{\mathrm{S}}_{\mathrm{zk}}$ & $1^{\text {st }}$ priority & $2^{\text {nd }}$ priority & $3^{\text {rd }}$ priority & Worst place \\
\hline$\widehat{\mathrm{S}}_{1 \mathrm{k}}$ & L4-2:+1.000 & L1-4:+0.761 & L1-2:+0.251 & L22-23:-0.379 \\
\hline$\widehat{\mathrm{S}}_{2 \mathrm{k}}$ & L28-13:+1.000 & L6-8:+0.336 & L22-7:+0.282 & L25-9:-0.084 \\
\hline$\widehat{\mathrm{S}}_{3 \mathrm{k}}$ & L26-12:+1.000 & L8-27:+0.722 & L27-12:+0.532 & L25-9:-0.062 \\
\hline$\widehat{\mathrm{S}}_{4 \mathrm{k}}$ & $\hat{L} 17-18:+1.000$ & L4-21:+0.027 & L1-21: +0.019 & L22-23:-0.042 \\
\hline$\widehat{\mathrm{S}}_{5 \mathrm{k}}$ & L25-9:+1.000 & L24-23:+0.714 & L24-25:+0.501 & L4-2:-0.067 \\
\hline
\end{tabular}

To verify the results, industrial software SIMPOW $R 11$ is used to simulate the test system in the presence of series compensators. Based on Table 3, it is clear that L26-12, L8-27 and L27-12 are the first priorities to install the series device for Fault 3 and L25-9 is the worst location. Fig. 4 shows the rotor angle of generator $11, \delta_{11}$ which is one of the most critical generators, for the same capacitive compensations of the mentioned lines. As it is expected, capacitive compensation of Line 26-12 has the best improvement of the transient stability while the same compensation of Line 25-9 worsens the transient stability. Then, a fixed series capacitance (in this study, equal to $50 \%$ compensation of each transmission line) has been placed in different locations and the new CCTs of the selected faults for most appropriate locations have been calculated by time domain simulations and given in Table 4 . It should be mentioned that the CCT values of faults for the system without compensation have been shown in Table 1 as well. It can be seen that the results are consistent with the normalized indices of trajectory sensitivities shown in Table 3 . The next step is to combine all the results and choose the most

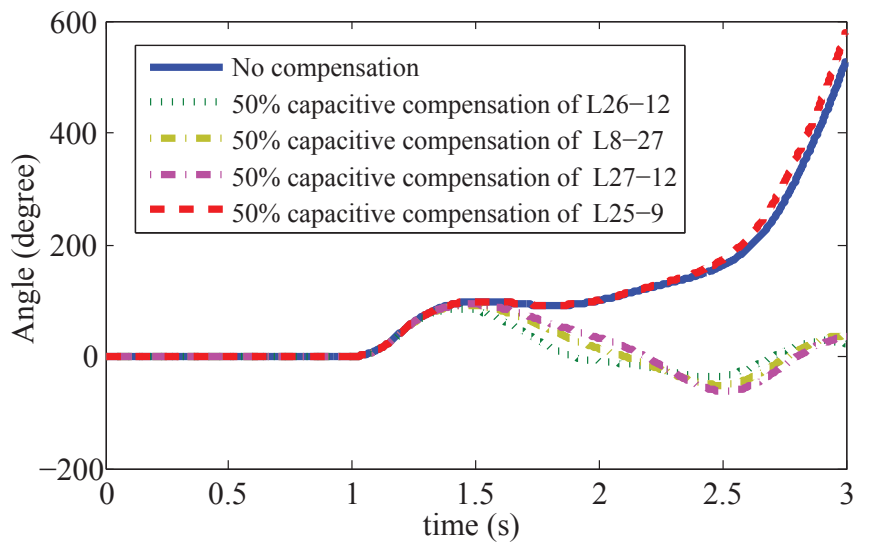

Fig. 4. Rotor angle of generator $11\left(\delta_{11}\right)$ for fault 3

TABLE IV

NEW CCTS OF THE FAULTS AFTER SERIES COMPENSATION OF THE MOST IMPORTANT LINES (MS)

\begin{tabular}{c|c|c|c|c}
\hline & $1^{\text {st }}$ priority & $2^{\text {nd }}$ priority & $3^{\text {rd }}$ priority & Worst place \\
\hline Fault 1 & L4-2: 175 & L1-4: 170 & L1-2: 168 & L22-23: 164 \\
\hline Fault 2 & L28-13: 243 & L6-8: 230 & L22-7: 228 & L25-9: 218 \\
\hline Fault 3 & L26-12: 215 & L8-27: 202 & L27-12: 199 & L25-9: 187 \\
\hline Fault 4 & L17-18: 316 & L4-21: 239 & L1-21: 239 & L22-23:237 \\
\hline Fault 5 & L25-9: 279 & L24-23: 274 & L24-25: 270 & L4-2: 264 \\
\hline
\end{tabular}

suitable place to install the first compensator. This decision is strongly dependent to the $p(z)$ function corresponding to each fault. For example, assuming the same probability of fault occurrence for difference scenarios and only considering the severity of each fault, $p(z)$ may have this structure

$$
p(z)=\frac{1}{C C T(z)} \quad \forall z \in Z
$$

This research does not intend to discuss about how to obtain the $p(z)$ function and (15) only recommends a way to incorporate the obtained data for all the fault scenarios and make the best decision about the final location to install the series device.

Considering the $p(z)$ structure introduced in (18) and using (15), the total trajectory sensitivity indices corresponding to different transmission lines, $\mathrm{TS}_{\mathrm{k}}$, have been calculated and are given in Table 5 for the most important lines. According

TABLE V

TOTAL TRAJECTORY SENSITIVITY INDICES TS $_{\mathrm{k}}$

\begin{tabular}{c|c|c|c|c}
\hline & $1^{\text {st }}$ priority & $2^{\text {nd }}$ priority & $3^{\text {rd }}$ priority & Worst place \\
\hline $\mathrm{TS}_{\mathrm{k}}$ & $\mathrm{L} 4-2:+6.182$ & $\mathrm{~L} 26-10:+5.071$ & $\mathrm{~L} 17-18:+4.451$ & L22-23:-1.3836 \\
\hline
\end{tabular}

to the Table 5, Line 4-2 is chosen as the most suitable place to install the first series compensator.

To install the next series device, new CCT values of selected faults in the presence of series compensator installed in Line 4-2 are computed and given in Table 6. As it was expected, the installed device only can improve the CCT value of fault 1 , and does not have any considerable effect on the other faults. 
TABLE VI

NEW CCT VALUES OF SELECTED FAULTS IN THE PRESENCE OF FIRST COMPENSATOR

\begin{tabular}{c|c|c|c|c|c}
\hline & Fault 1 & Fault 2 & Fault 3 & Fault 4 & Fault 5 \\
\hline CCT & $175 \mathrm{~ms}$ & $220 \mathrm{~ms}$ & $188 \mathrm{~ms}$ & $238 \mathrm{~ms}$ & $264 \mathrm{~ms}$ \\
\hline
\end{tabular}

Note that Line 4-2 is removed from the list of candidates to put the second device since it has already an installed device. The steps (1)-(3) of the propsed methodology described in Section 4 are repeated with the new situation of the system. Table 7 gives the new values of normalized indices of trajectory sensitivities $\widehat{\mathrm{S}}_{\mathrm{zk}}$ calculated for the placement of the second series compensator. The obtained data depicted in Table 7 determines the first three priorities to install the second series device and also the worst one for each fault. For the final TABLE VII

NORMALIZED INDICES OF TRAJECTORY SENSITIVITIES $\widehat{\mathrm{S}}_{\mathrm{zk}}-\mathrm{SECOND}$ DEVICE

\begin{tabular}{c|c|c|c|c}
\hline$\widehat{\mathrm{S}}_{\mathrm{zk}}$ & $1^{\text {st }}$ priority & $2^{\text {nd }}$ priority & $3^{\text {rd }}$ priority & Worst place \\
\hline$\widehat{\mathrm{S}}_{1 \mathrm{k}}$ & L1-4:+1.000 & L2-3:+0.368 & L1-2:+0.163 & L22-23:-0.432 \\
\hline$\widehat{\mathrm{S}}_{2 \mathrm{k}}$ & L28-13:+1.000 & L6-8:+0.336 & L22-7:+0.280 & L25-9:-0.083 \\
\hline$\widehat{\mathrm{S}}_{3 \mathrm{k}}$ & L26-12:+1.000 & L8-27:+0.715 & L27-12:+0.535 & L25-9:-0.064 \\
\hline$\widehat{\mathrm{S}}_{4 \mathrm{k}}$ & $\hat{L} 17-18:+1.000$ & L4-21:+0.026 & L1-5:+0.021 & L22-23:-0.032 \\
\hline$\widehat{\mathrm{S}}_{5 \mathrm{k}}$ & L24-42:+1.000 & L25-9:+0.899 & L24-23:+0.644 & L36-19:-0.034 \\
\hline
\end{tabular}

decision about the location of the second device, the $p(z)$ functions of each fault should be updated shown by $\widehat{p}(z)$. It is assumed that for the second device, the probability of fault occurrence is not similar and are according to the Table 8 .

TABLE VIII

PROBABILITY OF FAULT OCCURRENCE

\begin{tabular}{c|c|c|c|c|c}
\hline & Fault 1 & Fault 2 & Fault 3 & Fault 4 & Fault 5 \\
\hline$\overline{\mathrm{P}}(\mathrm{z})$ & $10 \%$ & $20 \%$ & $40 \%$ & $20 \%$ & $10 \%$ \\
\hline$\hat{\mathrm{p}}(\mathrm{z})=\frac{\overline{\mathrm{P}}(\mathrm{z})}{\mathrm{CCT}(\mathrm{z})} \quad \forall \mathrm{z} \in \mathrm{Z}$
\end{tabular}

The total trajectory sensitivity indices $\mathrm{TS}_{\mathrm{k}}$ for the second device are calculated using (19) and (15) and are given in Table 9. This table shows that with this definition of $\widehat{p}(z)$, Line 2612, Line 8-27 and Line 27-12 are the first priorities and Line $7-23$ is the worst location for the placement of second series compensator. These procedures can be repeated to allocate the next series devices.

TABLE IX

TOTAL TRAJECTORY SENSITIVITY INDICES $\mathrm{TS}_{\mathrm{k}}-\mathrm{SECOND}_{\mathrm{NEVICE}}$

\begin{tabular}{c|c|c|c|c}
\hline & $1^{\text {st }}$ priority & $2^{\text {nd }}$ priority & $3^{\text {rd }}$ priority & Worst place \\
\hline $\mathrm{TS}_{\mathrm{k}}$ & $\mathrm{L} 26-12:+2.102$ & $\mathrm{~L} 8-27:+1.565$ & $\mathrm{~L} 27-12:+1.098$ & L7-23:-0.158 \\
\hline
\end{tabular}

Regarding the computatioal burden, calculating these sensitivities based on the numerical formulation of TSA described in section II needs to run $\left(n_{L}+1\right) \times n_{z}$ time domain simulations to find the nominal and perturbed trajectories for a power system with $n_{L}$ lines (possible places to install series compensator) and $n_{z}$ fault scenarios. For this test system which has 52 lines and 5 fault scenarios, the required number of time domain simulations has been reduced from 265 $\left(\left(n_{L}+1\right) \times n_{z}\right)$ to $5\left(n_{z}\right)$ because of the analytical formulation of TSA. For the larger power systems, the proposed method simplifies significantly the cumbersome computational process of placement procedure.

\section{CONCLUSION}

A novel method has been proposed based on the trajectory sensitivity analysis (TSA) to find the suitable placement of multiple series compensators in order to improve the transient stability of power system. In this method, trajectory sensitivities of the rotor angles of the most critical generators with respect to the reactances of transmission lines for different fault scenarios have been computed. It has been clarified why putting series compensators in the transmission lines does not always improve the transient stability of power system. Using the analytical formulation of TSA and selecting appropriate system's parameters, the number of required time domain simulations have been reduced significantly. The numerical result on Nordic-32 test system have shown validity, accuracy and efficiency of the proposed approach.

\section{ACKNOWLEDGMENT}

Amin Nasri has been awarded an Erasmus Mundus $\mathrm{PhD}$ Fellowship. The authors would like to express their gratitude towards all partner institutions within the programme as well as the European Commission for their support.

\section{REFERENCES}

[1] G. A. Maria, C. Tang and J. Kim, "Hybrid transient stability analysis [power systems]," IEEE Trans. on power systems, vol. 5, no. 2, pp. 384-393, May 1990.

[2] A. A. Fouad, and S. E. Stanton, "Transient stability of a multi-machine power system. part ii. critical transient energy," IEEE Trans. on power appratus and systems, vol. PAS-100, no. 7, pp. 3417-3424, Jul. 1981.

[3] T. Athay, P. Podmore, and S. Virmani, "A practical method for the direct analysis of transient stability," IEEE Trans. on power appratus and systems, vol. PAS-98, no. 2, pp. 573-584, Mar. 1979.

[4] A. A. Fouad, V. Vittal, and T. K. Oh, "Critical energy for direct transeint stability assessment of a multimachine power system," IEEE Trans. on power appratus and systems, vol. PAS-103, no. 8, pp. 2199-2206, Aug. 1984.

[5] J. Zhao, A. Ishigame, S. Kawamoto, and T. Taniguchi "Structural control of electric power networks for transient stability," IEEE Trans. on power systems, vol. 9, no. 3, pp. 1575-1581, Aug. 1984.

[6] K. N. Shubhanga, and A. M. Kulkarni, "Application of structure preserving energy margin sensitivity to determine the effectiveness of shunt and series FACTS devices," IEEE Trans. on power systems, vol. 17, no. 3, pp. 730-738, Aug. 2002.

[7] A. Zamora-Cárdenas, and C. R. Fuerte-Esquivel, "Multi-parameter trajectory sensitivity approach for location of series-connected controllers to enhance power system transient stability," Electrical Power and Energy Systems, vol. 80, no. 9, pp. 1096-1103, Sep. 2010.

[8] M. J. Laufenberg, and M. A. Pai, "A new approach to dynamic security assessment using trajectory sensitivities," IEEE Trans. on power systems, vol. 13, no. 3, pp. 953-958, Aug. 1998.

[9] T. B. Nguyen, M. A. Pai, and I. A. Hiskens, "Sensitivity approaches for direct computation of critical parameters in a power system," International Journal of Electrical Power \& Energy Systems, vol. 24 no. 5, pp. 337-343, Jun. 2002.

[10] D. Chatterjee, and A. Ghosh, "Improvement of transient stability of power system with statcom-controller using trajectory sensitivity," Electrical Power and Energy Systems, vol. 33, no. 3, pp. 531-539, Mar. 2011.

[11] I. A. Hiskens, and M. A. Pai, "Trajectory sensitivity analysis of hybrid systems," IEEE Trans. circuits and systems, vol. 47, no. 2, pp. 204-220, Feb. 2000.

[12] M. Stubbe, "Long term dynamics phase ii, tf 38-02-08," Technical report, Cigre, pp. 1-19, Jun. 1995. 